The correlation between magneto-optical response and magnetic dipole resonance excitation in subwavelength silicon-nickel nanogratings

A. I. Musorin, M. G. Barsukova, A. S. Shorokhov, D. N. Neshev, Y. S. Kivshar, and A. A. Fedyanin

Citation: AIP Conference Proceedings 1874, 030026 (2017); doi: 10.1063/1.4998055

View online: http://dx.doi.org/10.1063/1.4998055

View Table of Contents: http://aip.scitation.org/toc/apc/1874/1

Published by the American Institute of Physics

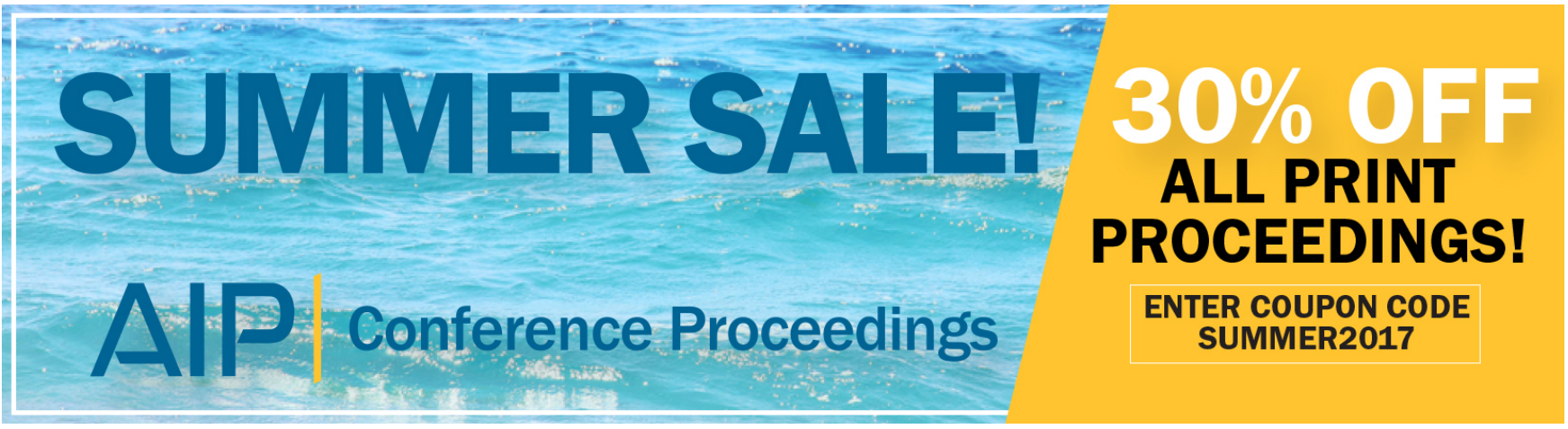




\title{
The Correlation Between Magneto-Optical Response and Magnetic Dipole Resonance Excitation in Subwavelength Silicon-Nickel Nanogratings
}

\author{
A.I. Musorin ${ }^{1}$, M.G. Barsukova ${ }^{1}$, A.S. Shorokhov ${ }^{1}$, D.N. Neshev ${ }^{2}$, Y.S. Kivshar ${ }^{2}$, \\ and A.A. Fedyanin ${ }^{1, \text { a) }}$ \\ ${ }^{1}$ Faculty of Physics, Lomonosov Moscow State University, Moscow 119991, Russia \\ ${ }^{2}$ Nonlinear Physics Centre, Research School of Physics and Engineering, Australian National University, Canberra \\ ACT 2601, Australia \\ ${ }^{\text {a)} C o r r e s p o n d i n g ~ a u t h o r: ~ f e d y a n i n @ n a n o l a b . p h y s . m s u . r u ~}$
}

\begin{abstract}
The advantages of gyrotopic materials are combined with the field of high-index metamaterials. The enhancement of the magneto-optical response in the spectral vicinity of the magnetic dipole resonance of a dielectric silicon nanodisks is numerically shown.
\end{abstract}

\section{INTRODUCTION}

Most of modern researches are devoted to the development of materials which allows manipulating the light characteristics, such as amplitude, phase or polarization [1]. Plasmonic nanostructures can localize electromagnetic field to the subwavelength scale. However, their efficiency for visible frequencies is limited due to large Ohmic losses in metals. New approaches are needed to control light on the nanoscale. Recent approaches towards alldielectric materials with high refractive index offer opportunities for design new nanophotonic materials [2].

One of the possibility to control light parameters is the use of external magnetic field. This method has several advantages, e.g. high speed of system response, no need to be in contact with the sample, lack of thermal influence etc. For the past few decades magneto-optical effects are usually observed in nanostructured hybrid materials or magnetoplasmonic crystals [3-5]. In this work a first step made to combine the advantages of all-dielectric highindex nanostructures and magnetic materials for creating compact active magneto-optical metadevices.

\section{SAMPLE}

The studied sample is an array of silicon disk-shaped nanoparticles. Silicon nanodisks, as one of the possible shapes, are attractive due to their resonance frequency adjusting capabilities. They have two degrees of freedom to manipulate: height and diameter. This opportunity allows one to tune the spectral position of magnetic and electric dipolar resonances. For example, their spectral overlapping leads to amplification of field localization and changing of scattering properties $[6,7]$. Here, the studied array of nanoparticles is placed on a quartz substrate to reach high contrast of refractive indices. A thin ferromagnetic film covers the system of disks and substrate.

\section{NUMERICAL CALCULATIONS}

The numerical calculations are performed with commercial software FDTD Solutions, Lumerical Inc. [8]. The optical and magneto-optical spectra are simulated. The distribution of electric and magnetic field for the spectral position of Mie-resonances is studied.

Proceedings of International Conference on Metamaterials and Nanophotonics (METANANO-2017)

AIP Conf. Proc. 1874, 030026-1-030026-2; doi: 10.1063/1.4998055

Published by AIP Publishing. 978-0-7354-1554-6/\$30.00 
The FDTD algorithm adapted to perform magneto-optical simulations is used. An elementary cell of the sample is modelled with periodic boundary conditions in $\mathrm{X}$, Y directions while in $\mathrm{Z}$ direction perfectly matched layers are used. A broadband plane wave source illuminates the structure under normal incidence. The polarization lies along $\mathrm{X}$ direction. The external magnetic field is directed along $\mathrm{Y}$ axis to simulate Voigt geometry, which correspond to intensity magneto-optical effect. The magneto-optical intensity effect is denoted by "MO response" and is defined as

$$
\text { MO response }=\frac{T(H)-T(0)}{T(0)},(1)
$$

where $\mathrm{T}(\mathrm{H})$ and $\mathrm{T}(0)$ are transmittance of the sample in case of existence and absence of magnetic field, respectively.

Figure 1 shows the reflectance (black line) and magneto-optical response (red line) spectra for one of the studied parameters of the structure. Numerical calculations show ten-fold enhancement of MO response around the Mieresonances of silicon disks in comparison with smooth ferromagnetic films deposited on quartz substrate with the same thickness of ferromagnetic metal (not shown on the figure).

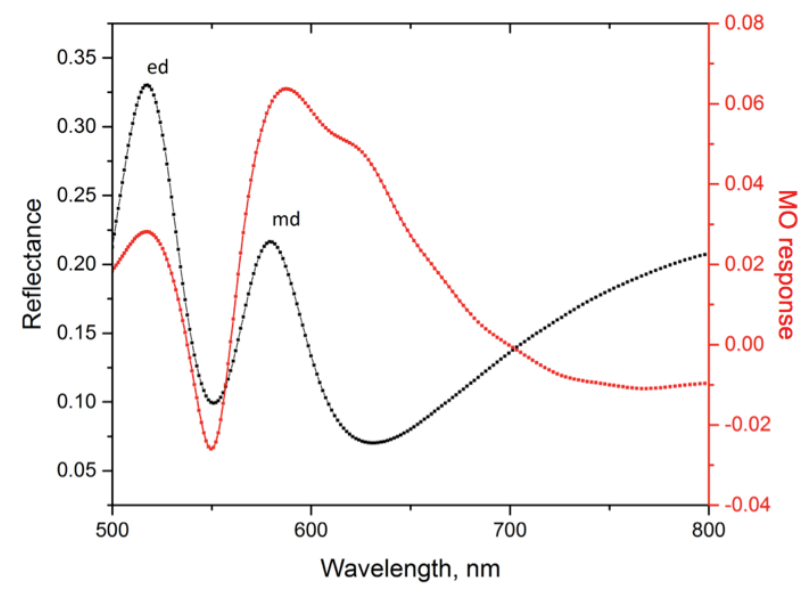

Figure 1. Reflectance (black) and TMOKE (red) spectra

\section{CONCLUSIONS}

In summary, we have numerically demonstrated the multifold enhancement of magneto-optical signal in vicinity of dipole Mie resonances of silicon nanodisks covered with a thin magnetic film. These results establish a novel approach for magnetic-field-controlled devices for silicon nanophotonics.

\section{ACKNOWLEDGMENTS}

The authors are grateful to M. Shcherbakov for fruitful discussions. This work was performed in Lomonosov Moscow State University and was supported by Russian Ministry of Education and Science (grant № 14. W03.31.0008).

\section{REFERENCES}

1. J. Saman and J. Zubin, Nature nanotech., 11, 23-36 (2016)

2. A.I. Kuznetsov, et al., Sci. Rep., 492, 1-6 (2012)

3. M. Inoue et al. "Magnetophotonics" in Springer Series in Materials Science (Springer-Verlag, Berlin Heidelberg, 2013), Chap. 178.

4. M. Inoue, et al. J. Phys. D. 39, R151 (2006)

5. L.E. Kreilkamp, et. al Phys. Rev. X, 3, 041019 (2013)

6. I. Staude, et al. ACS Nano, 7, 7824-7832 (2013)

7. Y.F. Yu, et al. Las. Photon. Rev., 9, 412-418 (2015)

8. http://lumerical.com/ 